\title{
Removal of Rectal Foreign Bodies Using Tenaculum Forceps Under Endoscopic Assistance
}

\author{
Keun Joon Lim, Joon Sung Kim, Boo Gyoung Kim, Sung Min Park, Jeong-Seon Ji, \\ Byung-Wook Kim, Hwang Choi \\ Department of Internal Medicine, The Catholic University of Korea, Incheon St.Mary's Hospital, Incheon, Korea
}

The incidence of rectal foreign bodies is increasing by the day, though not as common as that of upper gastrointestinal foreign bodies. Various methods for removal of foreign bodies have been reported. Removal during endoscopy using endoscopic devices is simple and safe, but if the foreign body is too large to be removed by this method, other methods are required. We report two cases of rectal foreign body removal by a relatively simple and inexpensive technique. A 42-year-old man with a vibrator in the rectum was admitted due to inability to remove it by himself and various endoscopic methods failed. Finally, the vibrator was removed successfully by using tenaculum forceps under endoscopic assistance. Similarly, a 59-year-old man with a carrot in the rectum was admitted. The carrot was removed easily by using the same method as that in the previous case. The use of tenaculum forceps under endoscopic guidance may be a useful method for removal of rectal foreign bodies. (Intest Res 2015;13:355-359)

Key Words: Rectum; Foreign bodies; Tenaculum forceps; Endoscopy

\section{INTRODUCTION}

The incidence of rectal foreign bodies is not known precisely but is occasionally encountered. ${ }^{1}$ Studies of rectal foreign bodies have suggested that most patients are men and aged 20 to 40 years old. ${ }^{2}$ Rectal foreign bodies are commonly inserted for anal erotic stimulation or gratification.

Many techniques for removal of rectal foreign bodies have been previously described. They can be removed during endoscopy with the assistance of endoscopic devices such as endoscopic snares or forceps. However, when foreign bodies cannot be removed by endoscopic devices, surgical methods may be needed. ${ }^{3}$ Transanal extraction is the main surgical

Received August 1, 2014. Revised October 13, 2014.

Accepted October 16, 2014.

Correspondence to Joon Sung Kim, Division of Gastroenterology,

Department of Internal Medicine, Incheon St. Mary's Hospital, The Catholic

University of Korea, 56 Dongsu-ro, Bupyeong-gu, Incheon 21431, Korea.

Tel: +82-32-280-5052, Fax: +82-32-280-5987,E-mail: kijoons@ catholic.

ac.kr

Financial support: None. Conflict of interest: None. procedure for removal; however, abdominal extraction may be needed in select cases. ${ }^{4}$

We report two cases of rectal foreign bodies removed by using tenaculum forceps under endoscopic assistance. We believe this method can be useful for foreign bodies that are too large to be removed by using an endoscope.

\section{CASE REPORT}

\section{Case 1}

A 42-year-old man was admitted to the emergency department because of inability to remove an adult product from his rectum. The patient described the incidence as his first event, and he had no specific history. The patient denied homosexual identity. No abdominal pain, nausea, or vomiting was observed. The patient's blood pressure was $128 / 82 \mathrm{~mm}$ Hg; pulse rate, $92 / \mathrm{min}$; respiration rate, $18 / \mathrm{min}$; and body temperature, $36.6^{\circ} \mathrm{C}$. Abdominal examination revealed a soft and flat abdomen with normal bowel sounds. Digital rectal

\footnotetext{
๑ Copyright 2015. Korean Association for the Study of Intestinal Diseases. All rights reserved.

This is an Open Access article distributed under the terms of the Creative Commons Attribution Non-Commercial License (http://creativecommons.org/licenses/by-nc/4.0)

which permits unrestricted non-commercial use, distribution, and reproduction in any medium, provided the original work is properly cited.
} 
examination revealed the hard plastic bottom of the object abutting the upper anal canal and normal anal sphincter tone. His laboratory data were as follows: hemoglobin level, $12.7 \mathrm{~g} / \mathrm{dL}$; white blood cell count, $8,940 / \mathrm{mm}^{3}$; and CRP level, $6.33 \mathrm{mg} / \mathrm{L}$. The results of evaluations for hepatitis B surface antigen, hepatitis $\mathrm{C}$ antibody, and human immunodeficiency virus (HIV) antibody were all negative. Radiography revealed a vibrator in the rectum, without evidence of peritoneal or perirectal free air (Fig. 1).

Several attempts to remove the vibrator by endoscopic snare, alligator forceps, grasping forceps, and u-turn technique with a transparent hood were made to no avail. As the endoscopic maneuvers failed to remove the foreign body, surgical removal under spinal anesthesia was planned. However, the patient refused to receive spinal anesthesia. We attempted to remove the foreign body by blindly using tenaculum forceps but could not grasp it tightly and failed (Fig. 2A). Finally, we decided to use the forceps under endoscopic as- sistance. After the endoscope was inserted in the anal canal, the tenaculum forceps was inserted and proceeded along the vision obtained by the endoscope until the distal ends reached the foreign body. Under direct endoscopic guidance, the tenaculum forceps was opened and used to grasp the end of the foreign body (Fig. 2B). Finally, the object was removed successfully. The removed object was relatively large and heavy (length, $25 \mathrm{~cm}$; diameter, $5 \mathrm{~cm}$; weight, 650 g). Mucosal injury was observed during endoscopic examination immediately after the removal. However, the patient did not complain of abdominal pain and therefore was discharged.

\section{Case 2}

A 59-year-old man was admitted to the emergency department with a carrot in the rectum and mild lower abdominal discomfort. He had no specific history and denied homo-
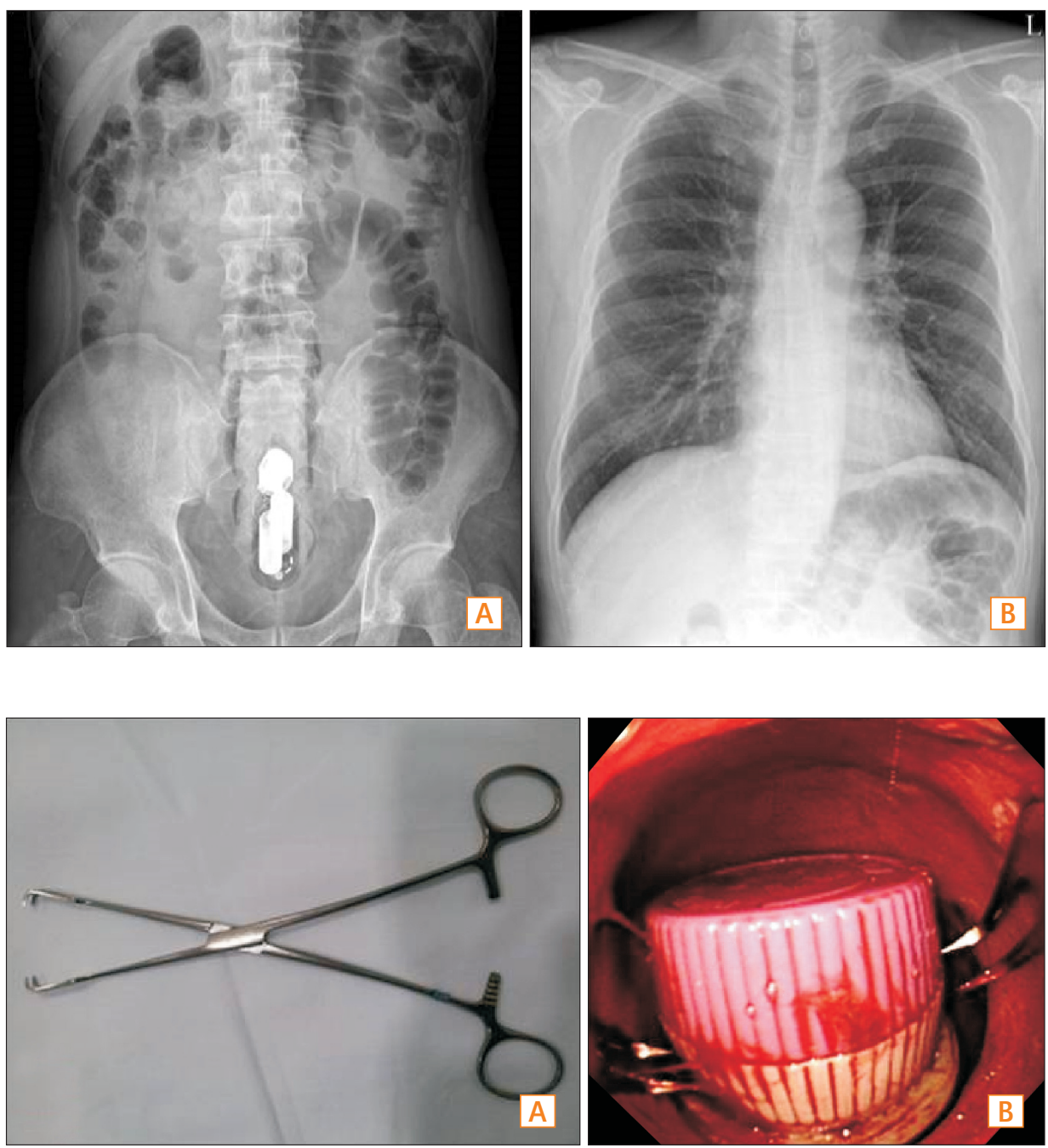

Fig. 2. Image of tenaculum forceps used in our case. (A) Tenaculum forceps used for foreign body removal. (B) A foreign body grasped by the tenaculum forceps under endoscopic assistance. 

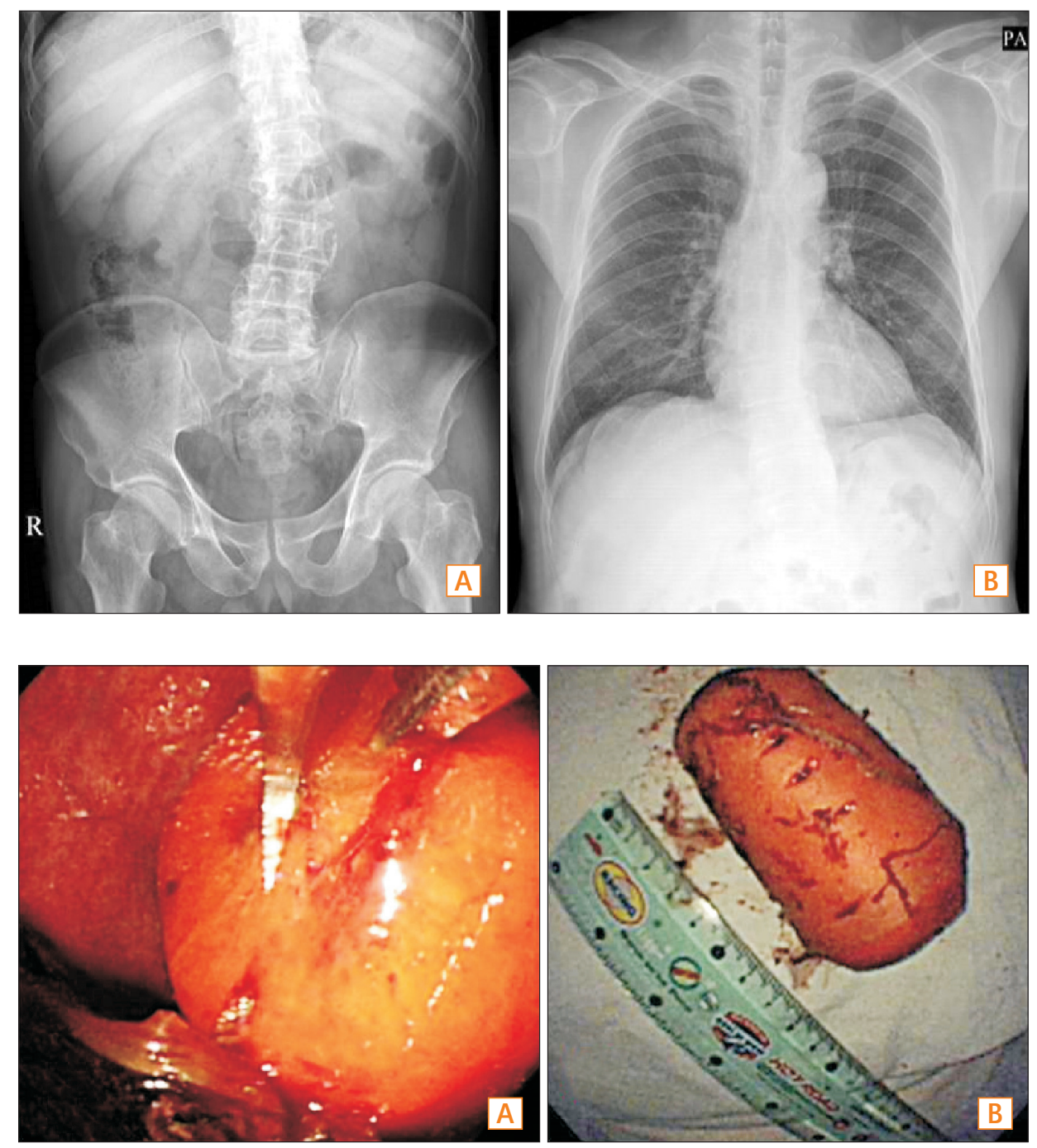

Fig. 3. Radiological findings of patients after removal of a rectal foreign body. (A) No foreign body in the rectum can be observed on the abdominal radiograph. (B) No evidence of abdominal free air can be observed on the chest radiograph.

Fig. 4. Image of foreign body removed in our case. (A) A foreign body grasped by tenaculum forceps under endoscopic assistance. (B) The removed foreign body (length, $8.5 \mathrm{~cm}$; weight, $200 \mathrm{~g}$ ).

sexual identity. The patient's blood pressure was 132/78 mm Hg; pulse rate, $88 / \mathrm{min}$; respiration rate, $18 / \mathrm{min}$; and body temperature, $36.5^{\circ} \mathrm{C}$. Abdominal examination revealed a soft and flat abdomen with normal bowel sounds. Digital rectal examination revealed the hard bottom of the carrot near the rectal angle and normal anal sphincter tone. His laboratory data were as follows: hemoglobin level, $14.7 \mathrm{~g} / \mathrm{dL}$; white blood cell count, 17,270/ $\mathrm{mm}^{3}$; and CRP level, $6.55 \mathrm{mg} / \mathrm{L}$. The results of evaluations for hepatitis B surface antigen, hepatitis $\mathrm{C}$ antibody, and HIV antibody were all negative. The foreign body was not observed on abdominal radiography, and no evidence of peritoneal or perirectal free air was observed (Fig. 3).

The carrot observed during endoscopy was deemed to be too large and heavy for removal using endoscopic devices. Hence, we decided to use tenaculum forceps from the start. By using the same techniques described previously, we were able to remove the carrot successfully (Fig. 4). The patient's abdominal discomfort subsided immediately, and he was discharged from the hospital.

\section{DISCUSSION}

Emergent endoscopy for removal of foreign bodies in the upper gastrointestinal tract is quite common, but foreign bodies in the rectum are not as common. However, the incidence of foreign bodies in the rectum has reportedly increased in recent decades, although no reliable epidemiological data are available. ${ }^{2,5}$ The main reason for insertion is autoeroticism, and patients can be HIV-positive in some cases. ${ }^{2,6,7}$ Initial laboratory tests should include HIV antibodies.

A thorough investigation as to the type of foreign object inserted is essential for deciding the removal method. ${ }^{5}$ If perforation caused by an irregular and sharp foreign body has occurred or is likely to occur during endoscopic removal, 
surgical methods for removal should be considered. ${ }^{6}$ In addition, patients with severe pain, fever, or peritoneal irritation signs should also undergo operation, as these suggest the possibility of perforation. ${ }^{6}$ However, operative intervention under anesthesia without these symptoms and signs entails great expense, so alternative nonsurgical removal methods are used in most cases. These include the use of peritoneum clamps, obstetric forceps, polypectomy snares, inflated Foley balloon catheters, and vacuum extractors. ${ }^{7-12}$

In the past, various grasping instruments, including tenaculum forceps, were used blindly in order to remove rectal foreign bodies and subsequent endoscopy after removal was performed to investigate mucosal injury. ${ }^{8,9}$ However, blind insertion of these instruments can hinder the removal of foreign bodies and can induce rectal injury or even perforation. Therefore, endoscopic removal would be a better and safer method. However, when the foreign body is too large, it may not be removed by simple endoscopic methods. In cases where endoscopic removal is impossible, transanal extraction under spinal anesthesia may be an alternative method. Spinal anesthesia helps to reduce the rectal tone and anal sphincter spasm, thereby improving chances of successful transanal retrieval. ${ }^{13}$ However, serious neurological complications after spinal anesthesia can occur rarely. ${ }^{14}$

In the first case, we tried to remove the foreign body by using various endoscopic instruments (snare, biopsy forceps, alligator forceps, and net) to no avail. Furthermore, the use of various devices prolonged the endoscopic time to more than an hour, and various attempts at removal resulted in injury to the rectal mucosa. Thus, we prepared the patient for spinal anesthesia, but the patient refused the procedure. Alternatively, we blindly used tenaculum forceps but had difficulty grasping the foreign object firmly without direct visualization. In addition, blind manipulation of the forceps could induce further mucosal injury. Finally, we removed the foreign body by using tenaculum forceps under endoscopic assistance. This method did not cause additional damage to the mucosa. The removed object was found to be too large and heavy for endoscopic removal.

Tenaculum forceps is a surgical instrument mostly used in obstetric surgery. It has a sharp-pointed hook at the end of each jaw and is used to grasp the anterior lip of the uterine cervix in total laparoscopic hysterectomy. In our cases, the hooks of the tenaculum forceps were useful for grasping the foreign body firmly and direct endoscopic guidance enabled us to grasp the foreign body precisely without any injury to the rectal mucosa.

In the second case, we used tenaculum forceps under endoscopic guidance from the beginning and removed the foreign body easily and safely within 5 minutes. Unlike firm objects such as a vibrator, relatively brittle objects such as carrots can be broken when grasped by forceps. In the second case, the distal end of the carrot was broken at the first attempt of grasping. Endoscopic assistance allowed us to grasp a larger part of the carrot on our second trial.

No specific criteria have been established regarding the specific size and weight of foreign bodies that can be removed by endoscopic devices. Use of endoscopic devices such as nets and snares are expensive and not covered by the Korean National Health Insurance. In addition, even if the foreign body is grasped by endoscopic devices, the curvature of the rectum may make removal difficult.

Our method is simple because only tenaculum forceps is used and is safe. Forceps other than tenaculum forceps can be used, but if the foreign body is lodged deep into the rectum, the length of the other forceps may be shorter. Indeed, in the first case, we used 16-cm-long Kelly hemostatic forceps and could not grasp the foreign body because the forceps was not long enough. After that, we used 25.5-cm-long tenaculum forceps, which allowed us to grasp the foreign body successfully. In addition, tenaculum forceps can widen up to $16 \mathrm{~cm}$ and has spike teeth of $0.7 \mathrm{~cm}$ in length, which enables the forceps to clutch the foreign body firmly.

We did not perform abdominal CT in both patients because their vital signs were stable and they showed no symptoms or signs of peritonitis. However, CT is important for documenting the position of the foreign object and ruling out perforation, and it should be considered before any manipulation. ${ }^{15}$ We planned to perform CT if symptoms and signs of peritonitis appeared, but no associated signs and symptoms were observed after removal.

Endoscopic removal in the case of large and heavy rectal foreign bodies is time-consuming and success can be difficult to achieve. In addition, in these patients, general or spinal anesthesia is a burden. Endoscopic removal using endoscopic devices should not be insisted in these circumstances. We present two instructive cases in which we safely and easily removed foreign bodies.

\section{REFERENCES}

1. Lake JP, Essani R, Petrone P, Kaiser AM, Asensio J, Beart RW, Jr. Management of retained colorectal foreign bodies: predictors of operative intervention. Dis Colon Rectum 2004;47:16941698. 
2. Ayantunde AA. Approach to the diagnosis and management of retained rectal foreign bodies: clinical update. Tech Coloproctol 2013;17:13-20.

3. Singaporewalla RM, Tan DE, Tan TK. Use of endoscopic snare to extract a large rectosigmoid foreign body with review of literature. Surg Laparosc Endosc Percutan Tech 2007;17:145-148.

4. Goldberg JE, Steele SR. Rectal foreign bodies. Surg Clin North Am 2010;90:173-184.

5. Smith MT, Wong RK. Foreign bodies. Gastrointest Endosc Clin N Am 2007;17:361-382.

6. Kasotakis G, Roediger L, Mittal S. Rectal foreign bodies: A case report and review of the literature. Int J Surg Case Rep 2012;3:111-115

7. Nivatvongs S, Metcalf DR, Sawyer MD. A simple technique to remove a large object from the rectum. J Am Coll Surg 2006;203:132-133.

8. Peet TN. Removal of impacted rectal foreign body with obstetric forceps. Br Med J 1976;1:500-501.
9. Levin SE, Cooperman H, Freilich M, Lomas M. The use of a curved uterine vulsellum for removal of rectal foreign bodies: report of a case. Dis Colon Rectum 1977;20:532-533.

10. Ahmed A, Cummings SA. Novel endoscopic approach for removal of a rectal foreign body. Gastrointest Endosc 1999; 50:872-874.

11. Steenvoorde P, Rovers JP, Tollenaar RA. Retained foreign body in a 14-year-old boy. J Pediatr Surg 2003;38:1554-1556.

12. Caliskan C, Karaca C, Akgun E, Korkut MA. A new extraction technique for rectal foreign bodies with a rubber band ligation device. Surg Today 2010;40:583-585.

13. Cirocco WC. Anesthesia facilitates the extraction of rectal foreign bodies. Gastrointest Endosc 2000;52:452-453.

14. Hyderally H. Complications of spinal anesthesia. Mt Sinai J Med 2002;69:55-56.

15. Jonnalagadda S, Edmundowicz SA. Percutaneous endoscopic gastrostomy and jejunostomy. In: Classen M, Tytgat GNJ, Lightdale CJ, eds. Gastroenterological endoscopy. 2nd ed. New York: Thieme, 2010:439-451. 